\title{
First comprehensive contribution to the knowledge of the lepidopteran fauna of Gioia Tauro Plain, South Italy (Lepidoptera)
}

\author{
D. Bonelli, ${ }^{1}$ S. Scalercio, ${ }^{2}$ T. Bonacci ${ }^{1}$ \\ ${ }^{1}$ Department of Biology, Ecology and Earth Sciences, University of Calabria, Rende (CS); ${ }^{2}$ Council for Agricultural \\ Research and Economics, Research Centre for Forestry and Wood, Rende (CS), Italy
}

\begin{abstract}
In this paper, we investigated for the first time on the composition of nocturnal Lepidoptera of the Gioia Tauro Plain, Calabria region (South Italy). The main goal was to acquire information on the biodiversity of this insect order in an area little investigated and subject to strong anthropic pressures. The study was carried out for one year in 4 sites characterized by different habitats. Sampling was carried out about twice a month through the use of UV-LED light traps. In total, 791 specimens belonging to 97 species were collected. The species richness of the study area was rather low compared to a more natural habitat
\end{abstract}

Correspondence: Domenico Bonelli, Department of Biology, Ecology and Earth Sciences. University of Calabria, via P. Bucci s.n., 87036, Rende (CS), Italy.

E-mail: domenico.bonelli@unical.it

Key words: Moth, Biodiversity, Italy, Anthropic impact.

Acknowledgements: We thank the Council for agricultural research and economics, Research Centre for Forestry and Wood in Rende, where it was possible to carry out bibliographic studies, compare the samples with the collections and use laboratories; another thank goes to Dr. Salvatore Bella (CREA - Acireale, Italy) for his help in determining Pterophoridae.

Contributions: The authors contributed equally.

Conflict of interest: The authors declare no potential conflict of interest.

Funding: None.

Received for publication: 21 January 2021

Revision received: 22 March 2021.

Accepted for publication: 18 May 2021.

${ }^{\circ}$ Copyright: the Author(s), 2021

Licensee PAGEPress, Italy

Journal of Entomological and Acarological Research 2021; 53:9632 doi:10.4081/jear.2021.9632

This article is distributed under the terms of the Creative Commons Attribution Noncommercial License (by-nc 4.0) which permits any noncommercial use, distribution, and reproduction in any medium, provided the original author(s) and source are credited. sampled near to the study area in previous years. However, we found species very interesting from a faunistic point of view. In detail, Morophaga morella (Duponchel, 1838) is a new record for the continental Italy. 15 species are new for the fauna of the Calabria region: Ornativalva tamaricella (Zeller, 1850), Cnephasia (Cnephasia) genitalana Pierce \& Metcalfe, 1915, Euzophera lunulella (O. Costa, 1836), Hellula undalis (Fabricius, 1794), Raphimetopus ablutella (Zeller, 1839) Achyra nudalis (Hübner, 1796), Dioryctria mendacella (Staudinger, 1859), Ematheudes punctella (Treitschke, 1833), Endotricha flammealis (Denis \& Schiffermüller, 1775), Oxybia transversella (Duponchel, 1836), Lamoria anella (Denis \& Schiffermüller, 1775), Anania crocealis (Hübner, 1796), Evergestis isatidalis (Duponchel, 1833), Udea ferrugalis (Hübner, 1796), and Uresiphita gilvata (Fabricius, 1794).

\section{Introduction}

In most of Mediterranean countries, there is a lack of faunistic data useful to evaluate long-term changes in ecosystems. The Mediterranean Basin is known to be very sensitive to changes due to climate and land use and the importance of biodiversity data assumes a key role to evaluate such changes especially in territories submitted to a strong human impact. The Gioia Tauro Plain, Calabria region (South Italy) is an example of these areas with a few data concerning biodiversity. During last centuries, this area has undergone many anthropogenic alterations causing landscape changes and the biodiversity loss as observed in similar cases in other areas (Ruberti \& Vigliotto, 2017). The sampled area is widely exploited at both agricultural and industrial levels reducing semi-natural vegetation to small patches along riversides and on steep slopes, the last ones occasionally burned.

The order of Lepidoptera is very species rich, changing community composition according to dominant vegetation, geographic gradient, and degree of human impact on ecosystems (Greco et al., 2018; Ienco et al., 2020). The ecological plasticity of this taxon is very useful for environmental and conservation studies (Sommaggio \& Paoletti, 2018). Several data are available for this insect order in many countries, but there are some areas where data are still very scarce or missing. Although during last years the knowledge on the lepidopteran fauna of Calabria region was strongly improved, many areas have never been investigated and the faunistic and ecological data on this group are not available. 
Faunistic data concerning Lepidoptera are very scarce and fragmented in the study area. In fact, only 31 macrolepidopteran species, mostly diurnal, are known for the Gioia Tauro Plain so far (see Annex 1), whilst more than 1,100 species are recorded at regional level (S. Scalercio, unpublished data) and 294 species have been recorded near the Angitola lake, the nearest locality submitted to methodic sampling of nocturnal macrolepidoptera (Scalercio \& Infusino, 2003). The first available data are very old, but few improvement of the species list occurred during last centuries (Petagna, 1786; Costa O.G., 1832-1836; Barrett, 1912; Stauder, 1915-1916; Stauder, 1923-1924; Lucchese, 1938; Verity, 1950-1951; Nardelli \& Giandolfo, 1994; Hensle, 2000; Longo et al., 2000; Balletto et al., 2005b; Flamigni et al., 2016), involving mostly diurnal Lepidoptera and only 3 microlepidoptera species (Trematerra et al. 2018). Among species recorded for the Gioia Tauro Plain only Melanargia arge (Sulzer, 1776) has a conservation interest being an endemic butterfly of peninsular Italy protected by the "Habitats Directive" $92 / 43 / \mathrm{CEE}$, and by the Berne Convention.

The aims of this study were (1) to assess the knowledge available in the study area for Lepidoptera, and (2) to improve the knowledge on the biodiversity of this important insect taxon in order to provide a georeferenced dataset useful to study changes that will likely occur next decades.

\section{Materials and methods}

\section{Study area}

The Gioia Tauro Plain extends for $243 \mathrm{~km}^{2}$. It is crossed by the Mesima River to the north and the Petrace River to the south (Figure 1); in the last two centuries the territory has been deeply transformed by human activities leading to the loss of even more rare ecosystems such as lowland forests and wetlands formerly widespread in the area (Gasparrini, 1840).

In the study area several infrastructures are presents such as the terminal container of the Port of Gioia Tauro, the Mediterranean Highway, several local roads, industries mainly linked to agricultural productions. Furthermore, urbanization involves agricultural lands strongly increasing the impact on semi-natural habitats menaced also by many pollution sources like industries, cities, dumbs, pollutants occasionally discarded directly into rivers. The strongly fragmented patches of semi-natural habitats are also menaced by the conversion in agricultural lands that is still occurring.

We chose 4 sampling sites representative of the natural habitat types present in the study area and located where fragmented seminatural vegetation are still present (Figures 1 and 2). This allowed us to collect species linked to different abiotic conditions increasing the representativeness of collected samples (Table 1).

Table 1. Sampling areas. For each site are reported identification code, the name of the locality, coordinates (DMS), altitude and habitat type.

\begin{tabular}{lcccc} 
Site & Locality-Municipality & Coordinates & Altitude (meters above sea level) & Habitat \\
A & Porta Sole - San Ferdinando & $38^{\circ} 29^{\prime} 32.30^{\prime \prime}$ N $15^{\circ} 55^{\prime} 0.40^{\prime \prime} \mathrm{E}$ & 7 & Dunal vegetation \\
B & Torre - San Ferdinando & $38^{\circ} 29^{\prime} 46.88^{\prime \prime} \mathrm{N} 15^{\circ} 55^{\prime} 4.23^{\prime \prime} \mathrm{E}$ & 5 & Retrodunal vegetation \\
\hline C & Mesima - Nicotera & $38^{\circ} 30^{\prime} 15.17^{\prime \prime} 15^{\circ} 55^{\prime} 27.77^{\prime \prime} \mathrm{E}$ & 4 & Riparian habitat \\
D & Torrente Serra - Cittanova & $38^{\circ} 20^{\prime} 42.19^{\prime \prime} 16^{\circ} 3^{\prime} 22.06^{\prime \prime} \mathrm{E}$ & 290 & Chestnut woodlot \\
\hline
\end{tabular}
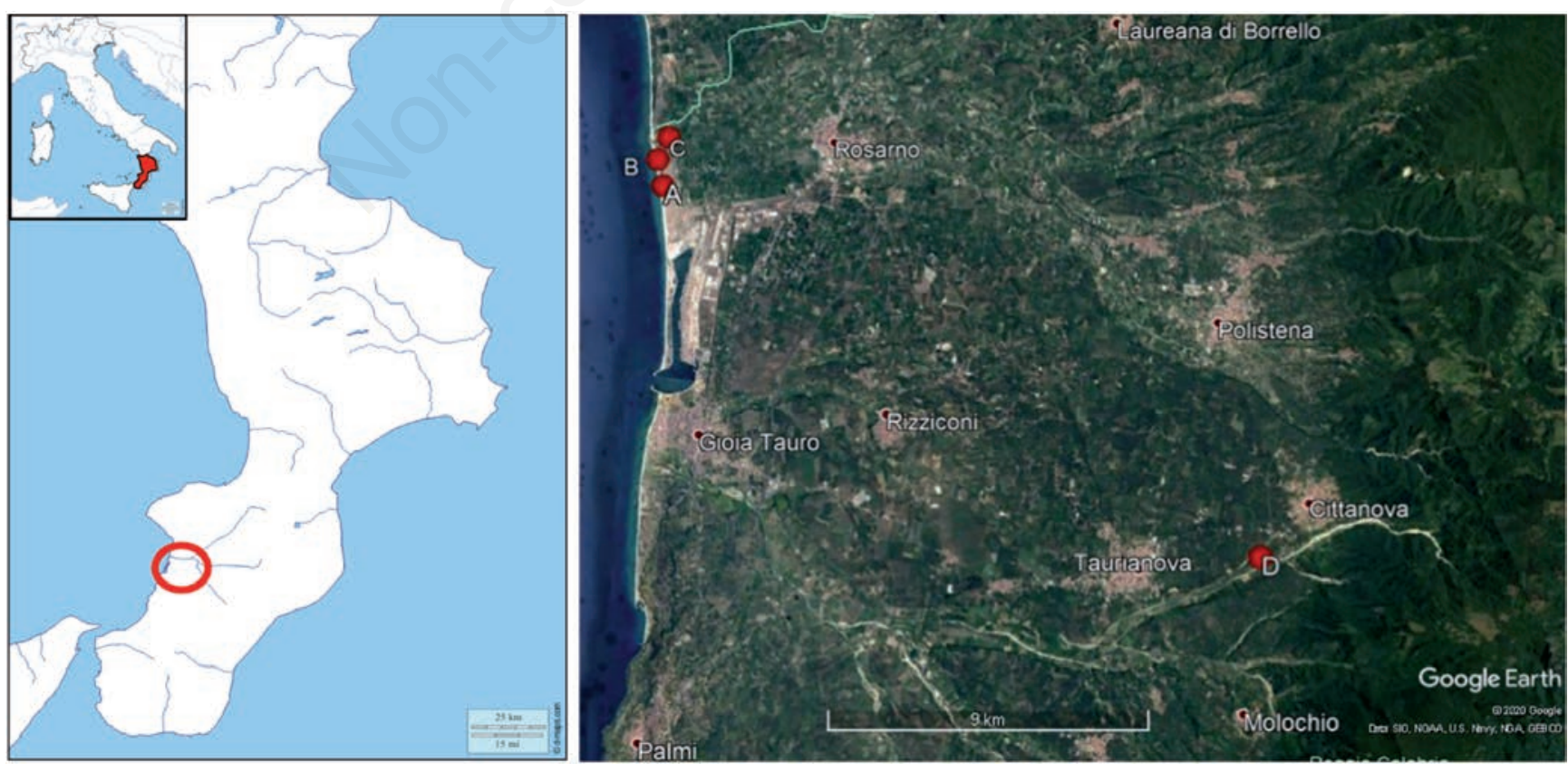

Figure 1. Study area. Geolocation of sampling areas (A, B, C and D). 
The site A is characterized by dunal and retrodunal vegetation that includes Retama raetam (Forssk.) Webb \& Berthel. subsp. gussonei (Webb) Greuter, found only in three Italian localities two of which are in Calabria (Caruso et al. 2010), and Ephedra fragilis Desf., found only here in Italian mainland (Caruso et al., 2012). Near this site a woodlot composed by Pinus pinea L., Acacia saligna (Labill.) H.L. Wendl., Eucalyptus camaldulensis Dehnh., and Tamarix africana Poir., is also present. Sparse bushes of Tamarix sp. divide dunal and retrodunal habitats. This site was sampled 20 times across the year (Figure 2A).

The site $\mathrm{B}$ is characterized by a retrodunal vegetation with the alien species Carpobrotus acinaciformis (L.) L. Bolus as dominant, and with the presence of Retama raetam gussonei and Ephedra fragilis. This site was sampled 20 times across the year (Figure 2B).

The site $\mathrm{C}$ represents a typical residual riparian habitat characterizing the flood plain strongly impacted by human activities such as deviation of the riverbed and water caption for agricultural activities. It is placed on the sandy bed of the Mesima River, near to a young woodlot of Salix sp. This site was sampled 4 times in August and September (Figure 2C).

The site D was in a hilly area characterized by a more mesophilous habitat composed by a small patch of Castanea sativa Mill., located about $20 \mathrm{~km}$ from coastal areas. This site was sampled only once in October (Figure 2D).

\section{Sampling}

The sampling period was from June 2017 to May 2018. The insects were sampled using UV-Led light traps (Infusino et al., 2017) activated from the sunset to the sunrise (Figure 3 ). The sampling was carried out for 45 nights, sampling twice per month during 2-4 consecutive nights. Our study was aimed to investigate on all macrolepidopteran assemblage, and only on few microlepidoptera species as for the latter taxonomic studies in Mediterranean countries are very rare and species identification is still uncertain, also using genetic studies. Unidentified material is available for further studies. Samples were sorted and specimens were identified in the entomological laboratory of the Research Centre for Forestry and Wood, Rende, where voucher specimens are included in the Lepidoptera research collection. Nomenclature followed the checklist reported in the Fauna Europaea website (https://fauna-eu.org/, last access 08/10/2020) (Karsholt \& Nieukerken, 2013).

Fifteen specimens were submitted to DNA barcoding, a technic based on the analysis of a $658 \mathrm{bp}$ sequence of the mitochondrial gene COI-1, largely used for species identification (Hajibabaei et al., 2006). Samples were prepared according to the protocol of the Canadian Center of DNA Barcoding, where samples were barcoded. Sequences are stored in the Barcoding of Life Database (BOLD) and compared to the other available in the database in order to confirm their correct identification, allowing us also to search for genetically isolated populations (Table 2).
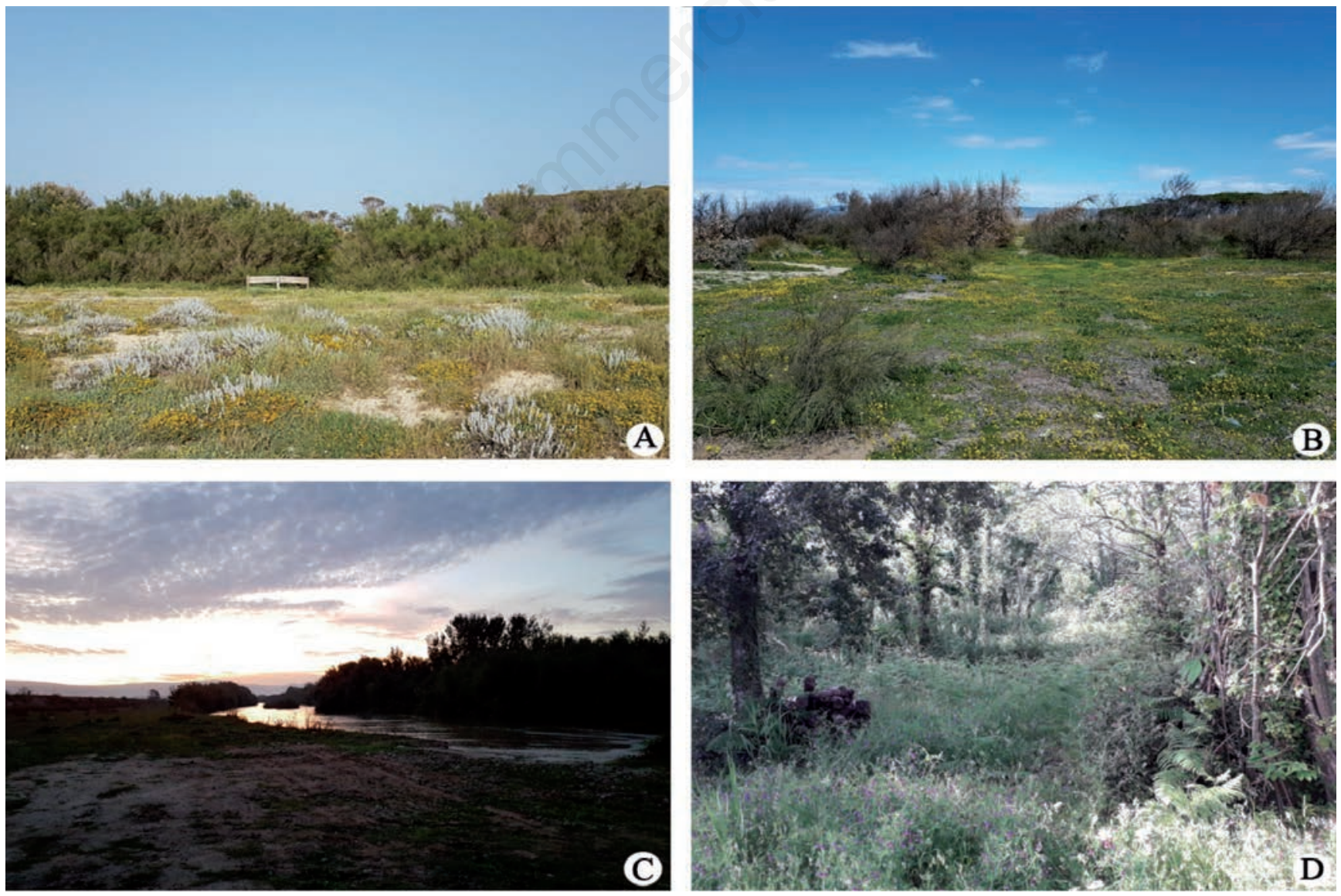

Figure 2. Habitat of the four sampled sites; A: dunal vegetation; B: retrodunal vegetation; C: riparian habitat; D: chestnut woodlot. 


\section{Results}

\section{Faunistic list}

A total of 792 individuals were identified, belonging to 16 families and 97 species as listed below. For each species we reported the site code, the sampling date, and the number of collected individuals. Species of faunistic relevance at national and regional level were indicated with an asterisk $(*)$.

\section{Psychidae}

Penestoglossa dardoinella (Millière, 1863) - B: 15.VII.2017 (1).

\section{Tineidae}

Morophaga morella (Duponchel, 1838) - A: 28.V.2018 (1), 30.V.2018 (2); B: 23.VI.2017 (2)

\section{Plutellidae}

Plutella (Plutella) xylostella (Linnaeus, 1758) - B: 26.VI.2017 (1).

\section{Gelechiidae}

*Ornativalva tamaricella (Zeller, 1850) - B: 17.V.2018 (1)

\section{Cossidae}

Parahypopta caestrum (Hübner, 1808) - A: 10.V.2018 (3), 13.V.2018 (5), 18.V.2018 (1), 26.V.2018 (1), 30.V.2018 (1).

\section{Tortricidae}

Aethes bilbaensis (Rössler, 1877) - A: 14.VII.2017 (1).

Aethes williana (Brahm, 1791) - A: 14.VII.2017 (1).

Cacoecimorpha pronubana (Hübner, 1799) - B: 15.VII.2017 (2).

* Cnephasia (Cnephasia) genitalana Pierce \& Metcalfe, 1915 - B: 13.V.2018 (1) (BARCODED).

Table 2. DNA Barcoding study of some collected specimens.

\begin{tabular}{llllc} 
Species & Data \& area & BOLD data & & \\
Specimen ID & Sequence ID & BIN \\
Cnephasia (Cnephasia) genitalana & $13 / 05 / 2018 \mathrm{~A}$ & LEP-SS-00911 & BCLEP461-18 & BOLD:AAC7742 \\
Apomyelois ceratoniae & $12 / 05 / 2018 \mathrm{~B}$ & LEP-SS-00890 & BCLEP439-18 & BOLD:AAU4812 \\
\hline Apomyelois ceratoniae & $20 / 05 / 2018 \mathrm{~B}$ & LEP-SS-00889 & BCLEP440-18 & BOLD:AAU4812 \\
Hellula undalis & $10 / 10 / 2017 \mathrm{~A}$ & LEP-SS-00862 & BCLEP412-18 & BOLD:AAC8519 \\
\hline Idaea longaria & 08/04/2018 A & LEP-SS-00806 & BCLEP356-18 & BOLD:ACE7056 \\
Dioryctria mendacella & $12 / 05 / 2018 \mathrm{~B}$ & LEP-SS-00841 & BCLEP391-18 & BOLD:ACF0860 \\
\hline Raphimetopus ablutella & $15 / 07 / 2017 \mathrm{~A}$ & LEP-SS-00883 & BCLEP433-18 & BOLD:ABV2048 \\
Lamoria anella & $13 / 05 / 2018 \mathrm{~A}$ & LEP-SS-00893 & BCLEP443-18 & BOLD:ACY8237 \\
\hline Lamoria anella & $18 / 05 / 2018 \mathrm{~A}$ & LEP-SS-00910 & BCLEP406-18 & BOLD:ACY8237 \\
Lamoria anella & $30 / 05 / 2018 \mathrm{~A}$ & LEP-SS-00894 & BCLEP444-18 & BOLD:ACY8237 \\
\hline Lamoria anella & $31 / 05 / 2018 \mathrm{~B}$ & LEP-SS-00912 & BCLEP462-18 & BOLD:ACY8237 \\
Lamoria anella & $12 / 05 / 2018 \mathrm{~B}$ & LEP-SS-00892 & BCLEP442-18 & BOLD:ACY8237 \\
\hline Lamoria anella & $12 / 05 / 2018 \mathrm{~B}$ & LEP-SS-00891 & BCLEP441-18 & BOLD:ACY8237 \\
Lamoria anella & $12 / 05 / 2018 \mathrm{~B}$ & LEP-SS-00895 & BCLEP445-18 & BOLD:ACY8237 \\
\hline Ematheudes punctella & $31 / 05 / 2018 \mathrm{~B}$ & LEP-SS-00913 & BCLEP463-18 & BOLD:ACP7474
\end{tabular}
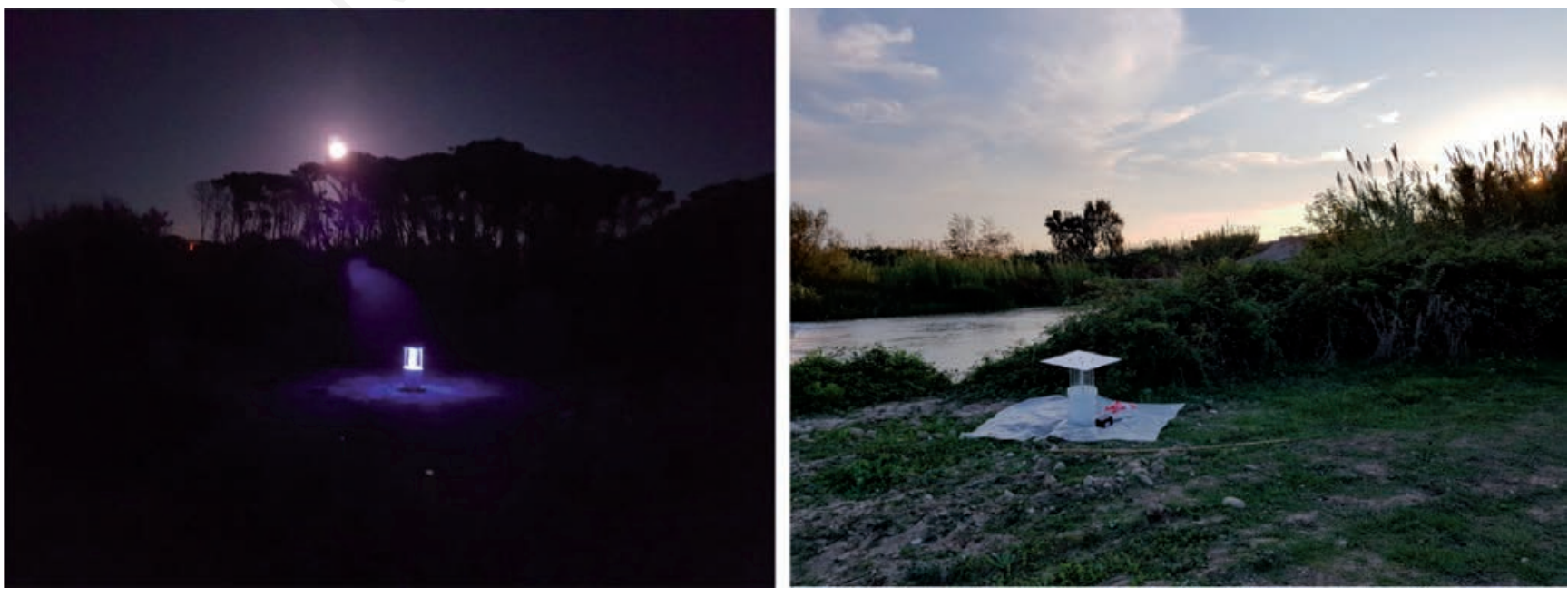

Figure 3. UV-LED light traps for capturing specimens. 


\section{Pterophoridae}

Agdistis tamaricis (Zeller, 1847) - A: 13.V.2018 (1) (det. Salvatore Bella).

\section{Pyralidae}

Apomyelois ceratoniae (Zeller, 1839) - B: 02. V.2018 (1) (BARCODED), 20.V.2018 (1) (BARCODED).

Dioryctria mendacella (Staudinger, 1859) - B: 12.V.2018 (1) (BARCODED).

Ematheudes punctella (Treitschke, 1833) - A: 25.IV.2017 (3), 14.IV.2017 (1), 18.V.2018 (1), 26.V.2018 (1), 28.V.2018 (1), 30.V.2018 (3); B: 03.VI.2017 (1), 26.VI.2017 (1), 02.V.2018 (1), 31.V.2018 (2) (BARCODED).

Endotricha flammealis (Denis \& Schiffermüller, 1775) - B: 02.V.2018 (1), 17.V.2018 (1).

Etiella zinckenella (Treitschke, 1832) - B: 15.VII.2017 (1).

Euzophera lunulella (O. Costa, 1836) - A: 02.VI.2017 (1), 25.VI.2017 (5), 26.V.2018 (1), 28.V.2018 (2), 30.V.2018 (1); B: 03.VI.2017 (1), 26.VI.2017 (11), 31.VI.2018 (1).

Hypotia corticalis (Denis \& Schiffermüller, 1775) - A: 02.VI.2017 (1), 25.VI.2017 (2); B: 31. VI.2018 (6).

Lamoria anella (Denis \& Schiffermüller, 1775) - A: 13.V.2018 (1) (BARCODED), 18.V.2018 (4) (BARCODED), 30.V.2018 (1) (BARCODED); B: 15.VII.2017 (1) (BARCODED), 12.V.2018 (3) (BARCODED), 17.V.2018 (3) (BARECODE), 31.V.2018 (1) (BARCODED).

Oxybia transversella (Duponchel, 1836) - A: 14.VII.2017 (5); B: 15.VII.2017 (1).

Raphimetopus ablutella (Zeller, 1839) - A: 14.VII.2017 (1) (BARCODED).

Pyralis farinalis (Linnaeus, 1758) - A: 18.V.2018 (1).

\section{Crambidae}

Achyra nudalis (Hübner, 1796) - A: 18.V.2018 (1); B: 02.V.2018 (1).

Anania crocealis (Hübner, 1796) - B: 15.VII.2017 (1).

Ancylolomia inornata Staudinger, 1870 - B: 15.VII.2017 (1), 02.V.2018 (1), 17.V.2018 (1).

Angustalius malacellus (Duponchel, 1836) - B: 02.V.2018 (1).

Duponchelia fovealis Zeller, 1847 - A: 18.V.2018 (1); B: 04.XI.2017 (1).

Eudonia angustea (Curtis, 1827) - A: 03.XI.2017 (4), 09.III.2018 (3), 22.IV.2018 (2), 28.IV.2018 (4).

Evergestis isatidalis (Duponchel, 1833) - A: 07.XII.2017 (1), 06.I.2018 (1).

Hellula undalis (Fabricius, 1794) - A: 25.VI.2017 (1), 10.X.2017 (1) (BARCODED);

B: 15.VII.2017 (1), 01.XI.2017 (1), 17.V.2018 (1).

Nomophila noctuella (Denis \& Schiffermüller, 1775) - A: 02.XI.2017 (1), 03.IV.2018 (1), 08.IV. 2018 (1), 10.V.2018 (1), 13.V.2018 (2), 18.V.2018 (3); B: 20. V.2018 (1).

Paracorsia repandalis (Denis \& Schiffermüller, 1775) - B: 29.IV.2018 (1), 02.V.2018 (1).

Udea ferrugalis (Hübner, 1796) - A: 03.IV.2018 (1), 08.IV.2018 (1); B: 10.III.2018 (1), 11.III.2018 (1), 29.III.2019 (1).

Uresiphita gilvata (Fabricius, 1794) - A: 02.VI.2017 (1), 14.VII.2017 (1), 13.V.2018 (1), 26.V.2018 (1), 28.V.2018 (1), 30.V.2018 (1); B: 15.VII.2017 (1), 12.V.2018 (1), 20.V. 2018 (1).

\section{Sphingidae}

Hyles livornica (Esper, 1780) - A: 02.VI.2017 (1).

Macroglossum stellatarum (Linnaeus, 1758) - A: 18.V. 2018 (1).

\section{Saturniidae}

Saturnia (Eudia) pavoniella (Scopoli, 1763) - B: 10.III.2018 (1).

\section{Drepanidae}

Habrosyne pyritoides (Hufnagel, 1766) - D: 21.X.2017 (1).

\section{Geometridae}

Ascotis selenaria (Denis \& Schiffermüller, 1775) - B: 15.VII.2017 (1).

Aspitates (Napuca) ochrearia (Rossi, 1794) - A: 10.X.2017 (5), 28.III.2018 (4), 03.IV.2018 (6), 08.IV.2018 (12), 22.IV.2018 (11), 24.IV.2018 (2), 28.IV.2018 (3); B: 06.X.2017 (1), 18. X.2017 (1), 10.III.2018 (2), 07.IV.2018 (4), 25.IV.2018 (5), 02.V.2018 (1); C: 15.IX.2017 (1).

Cabera exanthemata (Scopoli, 1763) - C: $20 . X .2017$ (1).

Cyclophora (Cyclophora) pupillaria (Hübner, 1799) - A: 25.VI.2017 (1); B: 26.VI.2017 (1); D: $21 . X .2017$ (1).

Epirrhoe alternata (Müller, 1764) - D: 21.X.2017 (1).

Eupithecia centaureata (Denis \& Schiffermüller, 1775) - A: 09.III.2018 (1).

Gymnoscelis rufifasciata (Haworth, 1809) - A: 02.VI.2017 (3), 25.VI.2017 (1), 14.VII.2017 (1), 30. V. 2018 (1); A2: 03.VI.2017 (1), 31.V.2018 (1).

Horisme radicaria (de La Harpe, 1855) - D: 21.X.2017 (1).

Idaea aversata (Linnaeus, 1758) - D: 21.X.2017 (18).

Idaea degeneraria (Hübner, 1799) - B: 25.IV.2018 (1), 17.V.2018 (1); D: 21.X.2017 (1).

Idaea distinctaria (Boisduval, 1840) - C: 15.IX.2017 (1).

Idaea elongaria (Rambur, 1833) - A: 08.IV.2018 (1), 26.V.2018 (4); B: 31.V.2018 (3).

Idaea filicata (Hübner, 1799) - A: $22 . I V .2018$ (7), 28.IV.2018 (1), 10.V.2018 (4), 13.V.2018 (1), 18.V.2018 (4); B: 25.IV.2018 (1), 02.V.2018 (1); C: 15.IX.2017 (10).

Idaea longaria (Herrich-Schäffer, 1852) - A: 08.IV.2018 (1) (BARCODED), 18.V.2018 (1), 28.V.2018 (2), 30.V.2018 (4); B: 20.V.2018 (1).

Idaea obsoletaria dionigii Hausmann, 1991 - A: 14.VII.2017 (1), 18.V.2018 (1); C: 15.IX.2017 (1).

Idaea rainerii Hausmann, 1994 - A: 18.V.2018 (1), 28.V.2018 (1); B: 03.VI.2017 (1).

Idaea rubraria (Staudinger, 1901) - D: 21.X.2017 (1).

Idaea rusticata (Denis \& Schiffermüller, 1775) - A: 25.VI.2017 (3). B: 26.VI.2017 (1).

Idaea seriata canteneraria (Boisduval, 1840) - A: 19.X.2017 (1), 09.III.2018 (1); B: 06.X.2017 (1), 18.X.2017 (1), 01.XI.2017 (2); C:15.IX.2017 (2).

Idaea subsericeata (Haworth, 1809) - A: 25.VI.2017 (1), 08.IV.2018 (1).

Ligdia adustata (Denis \& Schiffermüller, 1775) - D: 20.X.2017 (1).

Menophra abruptaria (Thunberg, 1792) - B: 26.VI.2017 (2).

Nycterosea obstipata (Fabricius, 1794) - A: 09.III.2018 (1), 18.V.2018 (1); B: 10.III.2018 (1), 19.V.2018 (1).

Pachycnemia hippocastanaria (Hübner, 1799) - A: 08.IV. 2018 (1).

Peribatodes rhomboidaria (Denis \& Schiffermüller, 1775) - A: 18.X.2018 (1); B: 26.VI.2017 (1), 15.VII.2017 (1), 06.X.2017 (2), 18.X.2017 (1).

Rhodometra sacraria (Linnaeus, 1767) - A: 10.X.2017 (2); B: 06.X.2017 (2); C: 15.IX.2017 (1).

Rhoptria asperaria (Hübner, 1817) - A: 24.IV.2018 (1); D: 21.X.2017 (1).

Scopula (Calothysanis) minorata (Boisduval, 1833)- A: 10.X.2017 (2); B: 29.III.2018 (1); C: 20.X.2017 (1).

Xanthorhoe disjunctaria (de La Harpe, 1860) - A: 06.I.2018 (1). 


\section{Notodontidae}

Clostera pigra (Hüfnagel, 1766) - B: 26.VI.2017 (1).

\section{Noctuidae}

Agrotis catalaunensis (Millière, 1873) - A: 02.XI.2017 (2), 03.XI.2017 (2), 06.III.2018 (6), 28.III.2018 (4), 03.IV.2018 (7), 22.IV.2018 (18), 26.IV.2018 (1); B: 06.X.2017 (2), 18.X.2017 (4), 01.XI.2017 (4), 10.III.2018 (11), 29.III.2018 (5), 07.IV.2019 (6), 25.IV.2018 (8).

Agrotis segetum (Denis \& Schiffermüller, 1775) - A: 25.VI.2017 (1), 07.XII.2017 (1).

Aporophyla (Aporophyla) australis (Boisduval, 1829) - A: 03.XI.2017 (2); B: 01.XI.2017 (1).

Aporophyla (Phylapora) nigra (Haworth, 1809) - D: 21.X.2017 (1).

Athetis (Proxenus) hospes (Freyer, 1831) - A: 02.VI.2017 (1), 25.VI.2017 (1), 14.VII.2017 (2); B: $26 . V I .2017$ (1), 02.V.2018

(1); C: 15.IX.2017 (1); D: 21.X.2017 (1).

Autographa gamma (Linnaeus, 1758) - A: 28.III.2018 (1).

Caradrina (Paradrina) clavipalpis Scopoli, 1763 - A: 22.IV.2018 (1); B: 25.IV.2018 (1).

Caradrina (Paradrina) flavirena Guenée, 1852 - A: 10.X.2017 (2), 19.X.2017 (1), 03.IV.2018 (2), 08.IV.2018 (6), 22.IV.2018 (5), 24.IV.2018 (3), 26.IV.2018 (1), 28.IV.2018 (1); B: 06.X.2017 (2), 18.X.2017 (2), 11.III.2018 (1), 07.IV.2018 (1), 02.V.2018 (1); C: 15.IX.2017 (7); D: 21.X.2017 (4).

Cryphia (Euthales) algae (Fabricius, 1775) - A: 25.VI.2017 (1).

Hadena (Anepia) silenes (Hübner, 1822) - A: 09.III.2018 (1), 08.IV.2018 (2); B: 25.IV.2018 (1), 02.X.2018 (2).

Hoplodrina ambigua (Denis \& Schiffermüller, 1775) - A: 10.X.2017 (1), 19.X.2017 (1), 22.IV.2018 (1), 26.IV.2018 (1); B: 18.X.2017 (2); C: 20.X.2017 (1).

Mormo maura (Linnaeus, 1758) - C: $20 . X .2017$ (1).

Mythimna (Pseudaletia) unipuncta (Haworth, 1809) - B: 25.IV.2018 (1).

Noctua comes Hübner, 1813 - B: 18.X.2017 (1).

Noctua janthina Denis \& Schiffermüller, 1775 - B: 26.VI.2017 (1).

Noctua pronuba (Linnaeus, 1758) - A: 19.X.2017 (1), 22.IV.2018 (1), 10.X.2018 (3); B: 06.X.2017 (1), 02.V.2018 (1); D: 21.X.2017 (1).

Spodoptera cilium Guenée, 1852 - C: $20 . X .2017$ (1).

Spodoptera exigua (Hübner, 1808) - A: 10.X.2017 (1), 02.XI.2017 (1), 26.V.2018 (1); C:20.X.2017 (1).

Xestia (Xestia) xanthographa (Denis \& Schiffermüller, 1775) - A: 19.X.2017 (1).

\section{Erebidae}

Arctia villica (Linnaeus, 1758) - A: 08.IV.2018 (1), 22.IV.2018 (1), 26.IV.2018 (2); B: 25.IV.2018 (1)

Clytie illunaris (Hübner, 1813) - A: 02.VI.2017 (2), 25.VI.2017 (1), 14.VII.2017 (2), 26.IV.2018 (1), 26.V.2018 (2); B: 03.VI.2017 (1), 26.VI.2017 (1), 15.VII.2017 (3), 02.V.2018 (1), 17.V.2018 (1), 19.V.2018 (1); C: $15 . I X .2017$ (1).

Cymbalophora pudica (Esper, 1784) - A: 10.X.2017 (3); B: 06.X.2017 (9), 18.X.2017 (8); C: 15.IX.2017 (1).

Dysauxes famula (Freyer, 1836) - A: 18.V.2018 (1), 26.V.2018 (1), 28.V.2018 (8), 30.V.2018 (1); B: 03.VI.2017 (2), 26.VI.2017 (1), 19.V.2018 (1), 31.V.2018 (5); C: $15 . I X .2017$ (1).

Eilema caniola (Hübner, 1808) - A: 02.VI.2017 (2), 14.VII.2017 (4), 10.X.2017 (5), 19.X.2017 (1), 10.V.2018 (6), 13.V.2018 (9), 18.V.2018 (12), 26.V.2018 (3); B: 03.VI.2017 (1), 26.VI.2017 (1), 06.X.2017 (3), 18.X.2017 (4), 01.XI.2017 (1), 02.V.2018 (6), 12.V.2018 (5), 17.V.2018 (9), 19.V.2018 (8), 20.V.2018 (2), 31.V.2018 (1); C: $15 . I X .2017$ (4); D: $21 . X .2017$ (2).
Eublemma ostrina (Hübner, 1808) - A: 22.IV.2018 (1); B: 17.V.2018 (2).

Eublemma parva (Hübner, 1808) - A: 14.VII.2017 (1), 10.X.2017 (1), 13.V.2018 (1), 18.V.2018 (2), 26.V.2018 (1), 28.V.2018 (1); B: 26.VI.2017 (1), 15.VII.2017 (1), 29.IV.2018 (4), 02.V.2018 (1), 17.V.2018 (1), 20.V.2018 (1), 31.V.2018 (3); C: 15.IX.2017 (1).

Hypena proboscidalis (Linnaeus, 1758) - D: 21.X.2017 (1).

Nodaria nodosalis (Herrich-Schäffer, 1851) - B: 06.X.2017 (1), 18.X.2017 (1), 04.XI.2017 (1); C: $20 . X .2017$ (4).

Phragmatobia fuliginosa (Linnaeus, 1758) - A: 25.VI.2017 (1), 09.III.2018 (1), 28.III.2018 (3), 03.IV.2018 (1), 08.IV.2018 (2), 22.IV.2018 (1); B: 26.VI.2017 (1), 06.X.2017 (1), 18.X.2017 (1), 10.III.2018 (2), 11.III.2018 (1), 29.III.2018 (1); C: 20.X.2017 (2).

Zebeeba falsalis (Herrich-Schäffer, 1839) - B: 01.XI.2017 (1); D: 21.X.2017 (1).

\section{Barcoding analysis}

Barcoding analysis involved 15 specimens belonging to 8 species (Table 2).

The sequence recovered for Ematheudes punctella is very common and identical to other 10 sequences deposited in BOLD. Perfect sequence identity also occurred in following species: Cnephasia (Cnephasia) genitalana, with three specimens from Croatia, Austria, and Germany; Hellula undalis with two specimens from Portugal, and Kenya; Dioryctria mendacella, with 3 specimens from Portugal, Spain, and Greece. The sequence of Idaea longaria is identical to those available for the Sicily, but quite different from those available for other geographic areas, allowing us to suppose the presence of a genetic lineage endemic of Sicily and Calabria regions corresponding to a BIN with a genetic distance of $1.61 \%$ (p-dist) from the Nearest Neighbor found in Spain (Figure 4).

The sequence recovered from Raphimetopus ablutella up to date appear to be endemic of the study area, diverging $0.61 \%$ from the nearest one from Greece.

We obtained more than one sequence only for Apomyelois ceratoniae $(\mathrm{n}=2)$ and Lamoria anella $(\mathrm{n}=7)$. In both species we found only two haplotypes, diverging the $0.16 \%$ in L. anella in which 5 specimens have the same haplotype, and diverging the $0.30 \%$ in $A$. ceratoniae. None of these haplotypes appears to be unique of the study area being recovered also from many other countries.

\section{Discussions and Conclusions}

The species richness of the study area is quite low if we compare macrolepidoptera founding a more natural habitat extensively surveyed in another Calabrian site, near the Angitola lake, located in similar climatic conditions (Scalercio \& Infusino, 2003). This partially depends on the different methods and on the different sampling effort of these studies, as the habitat alteration caused by human activities observed in the Gioia Tauro Plain had probably a major effect on species richness. However, we found some species very interesting from a faunistic point of view.

Morophaga morella is a new record for the Italian mainland; in Europe this species is known in all Mediterranean countries and in Ukraine; out of Europe is present in North Africa, Turkey, Caucasian and Afghanistan. The species lives in arid environments with sclerophyll formation where it feeds on various species of mushrooms and deadwood. The adults fly from May to October (Gaedike, 2015). 


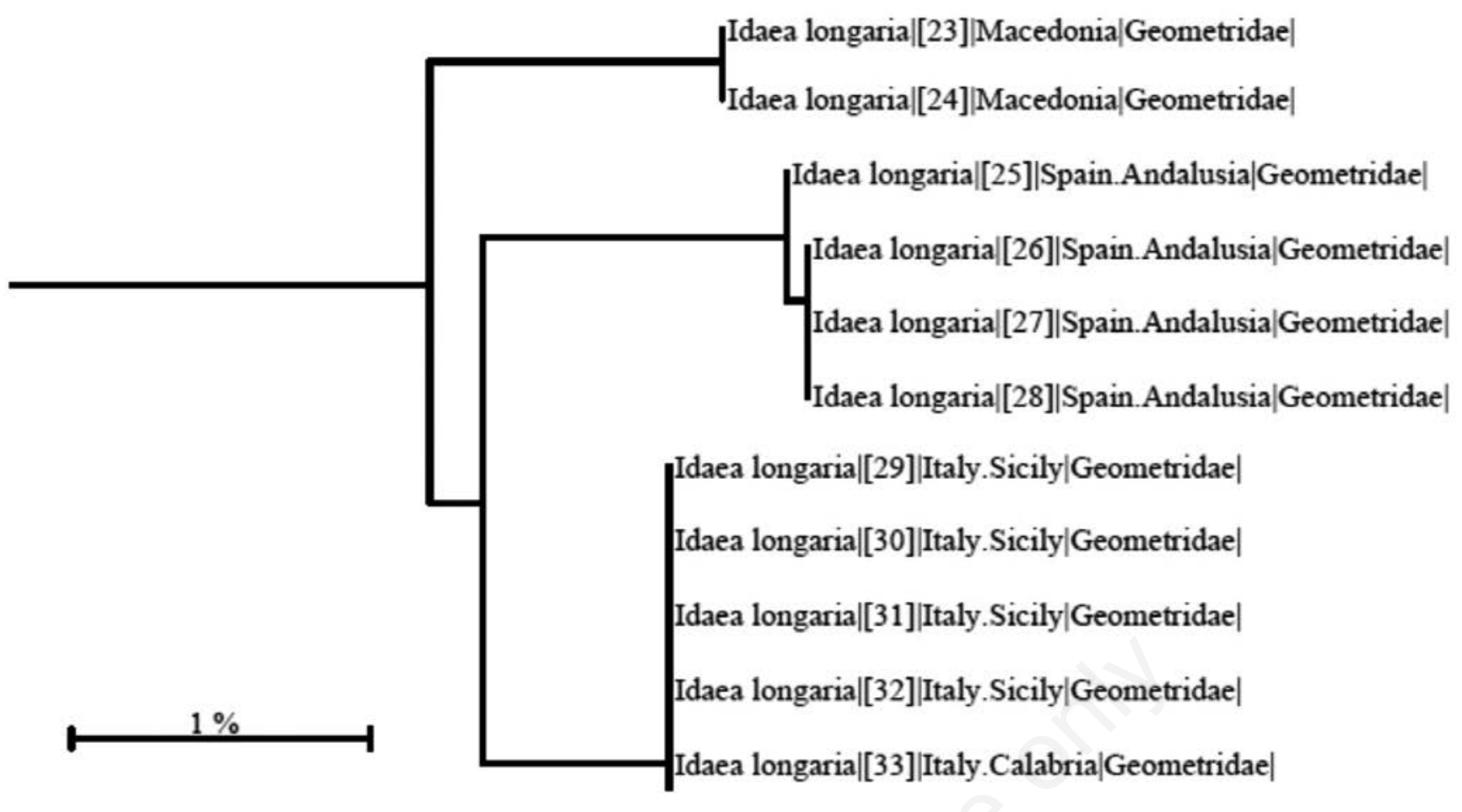

Figure 4. Neighbor-joining tree (Kimura 2-parameter distance model for COI-5P marker) for 11 specimens of the Idaea elongaria complex available in BOLD.

New records for Calabria are Ornativalva tamaricella known in Italy only for the Tuscany (Sattler, 1976); Cnephasia (Cnephasia) genitalana spread widely in Europe (Sweden, Spain) and North Africa; polyphagous species on various herbaceous plants. It is known in Italy only for Valle d'Aosta, Piedmont, Lombardy, Abruzzo, Molise, Campania, Basilicata and Sicily (Trematerra, 2003); Euzophera lunulella not record in Calabria so far; Hypotia corticalis is known in Italy only for the Vesuvio National Park (Vegliante \& Zilli, 2007); Hellula undalis spread widely, there are no records for Calabria (Vegliante \& Zilli, 2007); Raphimetopus ablutella is a new record for southern Italy, it is known only for North Italy and Sicily from where it was described (Bassi et al., 1995); Achyra nudalis generally known for most of Italian regions except the Alps (Slamka, 2013) and there are no records for Calabria; Dioryctria mendacella, Ematheudes punctella, Endotricha flammealis, Oxybia transversella, Lamoria anella, Anania crocealis, Evergestis isatidalis, Udea ferrugalis, and Uresiphita gilvata reported generally for southern Italy (Bassi et al. 1995), but there are no records for Calabria; Idaea longaria known only in coastal areas of Sicily and Latium so far (Hausmann, 2004); Idaea rainerii recorded for the second time in Calabria, for the first time recorded at the $\mathrm{La}$ Vota lakes (Gizzeria, Catanzaro) (Parenzan et al., 1998).

Idaea longaria, found for the first time in Calabria in this study, and I. rainerii are both interesting also from an ecological point of view. In fact, these species live in sandy and brackish coastal habitats with halophilic vegetation (Hausmann, 2004). Around the Mediterranean Basin, these habitats are really threatened by the human activities that increased their fragmentation. The loss of these habitats causes the local rarefaction or extinction of these species and for this reason, their conservation is extremely important. This appear to be of particular importance for the population of I. longaria that belong to a genetic lineage endemic of Sicily and Calabria regions.
During next years, we will continue to study the lepidopteran diversity of the Gioa Tauro Plain increasing sampling effort in under-investigated habitats, as chestnut woodlots and riparian habitat are in this study, and increasing the identification effort concerning microlepidoptera. We expect that more deep sampling and taxonomic efforts will reveal a richer lepidopteran fauna, providing a useful dataset for investigating the effects of long-term ecological drivers on local and Mediterranean biodiversity.

\section{References}

BALLETTO E., BONELLI S., CASSULO L., 2005 - Insecta Lepidoptera Papilionoidea. In: Ruffo S., Stoch F. (eds.), Checklist e distribuzione della fauna italiana. 10.000 specie terrestri e delle acque interne. - Mem. Mus. Civ. Stor. Nat. Verona - 2. Serie, Sezione Scienza della Vita 16: 259-263, 309 pp.

BARRETT J.P., 1912 - Butterfly collecting in Sicily and Calabria in 1911 - Entomologist, XLV: 303-306.

BASSI G., PASSERIN D'ENTREVÈS P., SPEIDEL W., ZANGHERI S., 1995 - Lepidoptera Pyraloidea. In MINELLI A., RUFFO S. \& LA POSTA S. (eds.). Checklist delle specie della fauna italiana, 87. Calderini, Bologna.

CARUSO G., GANGALE C., SCIANDRELLO S., UZUNOV D., 2010 - Retama raetam (Forsskl.) Webb et. Berthel. subsp. gussonei (Webb) Greuter. - Inf. Bot. Ital., 42: 539- 613.

CARUSO G., UZUNOV D., GANGALE C., PIGNOTTI L., 2012 - Notes on the distribution of the genus Ephedra L. (Ephedraceae) in Calabria (S Italy). - Bot. Serb. 36: 15-21.

COSTA O.G., 1832-1836 - Fauna del Regno di Napoli. Lepidotteri. Parte prima. Lepidotteri Diurni, Crepuscolari ed 
alcune famiglie de' Notturni. - Napoli, dai torchi del Tremater, pp. XI + 204, tavv. I-XIV.

FLAMIGNI C., FIUMI G. \& PARENZAN P., 2016 - Lepidotteri Eteroceri d'Italia. Geometridae Ennominae II. Natura Edizioni Scientifiche, Bologna, 512 pp, 32 tavv.

GAEDIKE R., 2015 - Tineidae I (Dryadaulinae, Hapsiferinae, Euplocaminae, Scardiinae, Nemapogoninae and Meessiinae). Microlepidoptera of Europe, 7. Brill NV,Netherlands, 308 pp.

GASPARRINI G., 1840 - Discorso intorno all'origine del villaggio di San Ferdinando e sopra le principali cose che quivi si coltivano. s.n., Napoli, 113 pp.

GRECO S., INFUSINO M., IENCO A., SCALERCIO S., 2018 How different management regimes of chestnut forests affect diversity and abundance of moth communities? - Ann. Silvic. Res. 42: 59-67.

HAJIBABAEI, M., JANZEN, D.H., BURNS, J.M., HALLWACHS, W., HEBERT, P.D.N., 2006 - DNA barcodes distinguish species of tropical Lepidoptera. - Proc. Natl. Acad. Sci. USA, 103: 968-971.

HAUSMANN A., 2004 - Sterrhinae. - In A. Hausmann (ed.): The Geometrid Moths of Europe. Vol. 2: 1-600. Apollo books, Stenstrup, Denmark.

HENSLE J., 2000 - Herbstfunde von Danaus chrysippus (Linnaeus, 1758) in Kalabrien (Süditalien) (Lepidoptera, Nymphalidae, Danainae). - Atalanta, 31 (1/2): 56-60.

IENCO A., DAPPORTO L., GRECO S., INFUSINO M., SCALERCIO S., 2020 - $\beta$-Diversity partitioning of moth communities within and between different forest types. - Sci. Nat. 107: 8 .

INFUSINO M., BREHM G., DI MARCO C., SCALERCIO S., 2017 - Assessing the efficiency of UV LEDs as light sources for sampling the diversity of macro-moths (Lepidoptera) - Eur. J. Entomol. 114: 25-33.

KARSHOLT, O., NIEUKERKEN, E.J. VAN, 2013 - Lepidoptera, Moths. Fauna Europaea version 2017.06, https://fauna-eu.org.

LONGO S., PALMERI V., CAROLEI A.E., 2000 - Biologia di Charaxes jasius in agrumeti della Calabria (Lepidoptera Nymphalidae). - Boll. Soc. Entomol. Ital. 132: 83-90.

LUCCHESE E., 1938 - Contributi alla conoscenza dei Lepidotteri del Melo. II. Aegeria typhiaeformis Bkh. - Bollettino del Laboratorio di Zoologia Generale e Agraria, Portici, XXXI: 158-195.

NARDELLI U., GIANDOLFO B., 1994 - Melanargia pherusa Boisduval, 1833: Biologie, Ökologie, Morphologie und Verbreitung im Vergleich mit Melanargia arge Sulzer, 1776 (Lepidoptera: Nymphalidae, Satyrinae). - Nachrichten des entomologischen Vereins Apollo, N. F., 15: 87-108, 1 cartina, 4 tavv., 7 figg.
PARENZAN P., HAUSMANN A., SCALERCIO S., 1998 Addenda e corrigenda ai Geometridae dell'Italia meridionale (Contributi alla conoscenza della Lepidotterofauna dell'Italia meridionale. XX - Entomologica 32: 51-79.

PETAGNA V., 1786 - Specimen Insectorum ulterioris Calabriae. Neapoli, Typis Petri Perger, pp. I-VII, 1-45, 1 Tav.

RUBERTI D., VIGLIOTTO M., 2017 - Land use and landscape pattern changes driven by land reclamation in a coastal area: the case of Volturno delta plain, Campania Region, southern Italy. - Environ. Earth Sci. 76: 694.

SATTLER, K., 1976 - A taxonomic revision of the genus Ornativalva Gozmány, 1955 (Lepidoptera: Gelechiidae). - Bull. Br. Mus. Nat. Hist. Entomol. 34: 85-152.

SLAMKA F., 2013 - Pyraloidea (Lepidoptera) of Europe, Volume 3 Pyraustinae \& Spilomelinae. Frantisek Slamka, Slovakia, 357 pp.

SOMMAGGIO D., PAOLETTI M.G., 2018 - Gli invertebrati come bioindicatori di un paesaggio sostenibile. Edizioni Libreria universitaria.it, Roma, 278 pp.

SCALERCIO S., INFUSINO M., 2003 - I Macrolepidotteri di Fosso Scuotrapiti, lago dell'Angitola (Calabria, Italia meridionale) (Lepidoptera). - Phytophaga, 13: 25-52.

STAUDER H., 1915-1916 - Lepidopteren aus dem Aspromontegebirge. Material zu einer Zusammenstellung der südkalabrischen Schmetterlingsfauna. - Zeitschrift für wissenschaftliche Insektenbiologie, XI (1915) (11-12): 281-286; XII (1916) (1-2): 10-14; (3-4): 59-63; (5-6): 109-112, 1 Taf.

STAUDER H., 1923-1924 - Lepidopteren aus Unteritalien. I. Societas entomologica, 38 (1923) (2): 5-7, (3): 10-11, (4): 1416, (5): 19-20, (6): 23-24, (7): 27-28, (8): 30-32, (9): 35-36, (10): 37-40, (11): 43-44, (12): 46-48, 39 (1924) (1): 3-4, (2): $7-$ 8, (3): 10-12, (4): 15-16, (5): 19-20, (6): 23-24, (7): 26-28, (8): 29-31, (9): 34-35, (10): 38-39.

TREMATERRA P., 2003 - Catalogo dei Lepidotteri Tortricidae della fauna italiana: geonemia, distribuzione in Italia, note biologiche, identificazione. - Boll. Zool. agr. Bachic., Ser. II, 35: 1-270.

TREMATERRA P., GOGLIA L., SCALERCIO S., COLACCI M., 2018 - Lepidoptera Tortricidae from Calabria (Southern Italy). - Redia, 101: 167-181.

VEGLIANTE F., ZILLI A., 2007 - The butterflies and moths of the park and surroundings (Lepidoptera). Artropodi del parco nazionale del Vesuvio: ricerche preliminari. - Conservazione Habitat Invertebrati, 4/2007.307-364.

VERITY R., 1950-1951 - Le Farfalle Diurne d'Italia. Vol. IV. Papilionida (Libytheina, Danaina e Nymphalina: Apaturidae e Nymphalidae). - Ed. Marzocco, Firenze, XXXV + 380 pp. (1950), Tavv. 38-54 + XV-XX (1951). 